Hausarztmedizin, Dr. Jürg Bleuer, Leiter der EBM-Abteilung des DOKDI, Dr. Beat Künzi von der Schweizerischen Gesellschaft für Allgemeinmedizin sowie Dr. Hermann Amstad und seinen Mitarbeiterinnen vom Generalsekretariat der Akademie herzlich danken.

Die grosse Zahl von Teilnehmerinnen und Teilnehmern am heutigen Symposium zeigt die Bedeutung, die dem Thema Evidence-based Medicine und Guidelines von der Ärzteschaft beigemessen wird. Ich hoffe, dass die Referate und Diskussionen dazu bei- tragen werden, EBM und Guidelines als das zu sehen, was sie sein sollen: ein Instrument in der Hand des Arztes zum Wohl des Patienten.

\footnotetext{
Literatur

1 Rosenbaum S, Frankord DM, Moore B, Borzi P. Who should determine when health care is medically necessary? N Engl J Med 1999;340:229-32.
}

\section{EBM - ein Paradigmenwechsel?}

\author{
J. P. Bleuer
}

\section{Zusammenfassung}

Die Vielfalt an Stossrichtungen hat Evidence-based Medicine (EBM) insbesondere zu Beginn Kritik von verschiedenster Seite eingebracht und zu heftigen Diskussionen geführt. So wurde und wird EBM vorgeworfen, es sei ein alter Hut, in der Medizin hätte man schon immer nach bester Evidenz gehandelt. Einerseits trifft der Vorwurf nicht zu; dies lässt sich bereits aus der Vielfalt parallel existierender therapeutischer und diagnostischer Prozedere bei gleicher Problemstellung ableiten. Anderseits verkennt er auch, dass Evidence-based Medicine das Wort «evidence" in der englischsprachigen Bedeutung von "Beweis" verwendet und damit fordert, die Richtigkeit von Lehrmeinungen seien prinzipiell zu belegen, wenn immer möglich auf der Basis klinisch-epidemiologischer Daten. Der hohe Stellenwert epidemiologischer Daten leitet sich ab aus der Grundhaltung der EBM, die nicht Krankheitsphänomene mit naturwissenschaftlichen Methoden analysieren, sondern die Zweckmässigkeit von Interventionen belegen

Korrespondenz:

Dr. med. Jürg P. Bleuer

Abteilungsleiter Evidence-based Medicine

Dokumentationsdienst der Schweizerischen Akademie

der Medizinischen Wissenschaften DOKDI

Postfach 5921

CH-3001 Bern

E-mail: bleuer@sams.ch will. EBM hinterfragt bisher akzeptierte diagnostische Tests und therapeutische Verfahren, um sie bei Bedarf durch effektivere und sicherere zu ersetzen.

\section{Zum Paradigmenwechsel}

Thomas Kuhn bezeichnete das Paradigma als «das allgemein akzeptierte Vorgehen einer wissenschaftlichen Disziplin", und Bortz und Döring schreiben in ihrem Buch «Forschungsmethoden und Evaluation» über den Paradigmenwechsel: "Eingeleitet wird dieser Prozess durch das Auftreten von Befunden, die es eigentlich nicht geben dürfte" [1]. EBM plädiert in der Tat aufgrund des "Auftretens von Befunden, die es eigentlich nicht geben dürfte», für einen Wechsel im "allgemein akzeptierten Vorgehen». Ob deshalb bereits von einem Paradigmenwechsel zu sprechen ist, soll im folgenden diskutiert werden.

\section{Befunde, die es eigentlich nicht geben dürfte}

Unter den Befunden, die es eigentlich nicht geben dürfte, ist die Vielfalt parallel existierender therapeutischer Vorgehensweisen bei gleicher Problemstellung augenfällig. Ein Beispiel dafür ist die Otitis media bei Kindern: Während in den Niederlanden initial meist keine Antibiotika gegeben werden, waren diese noch 1997 in den anderen industrialisierten Ländern Standard; in den Vereinigten Staaten ist Otitis media sogar die häufigste Indikation für eine Antibiotikagabe im ambulanten Bereich. Ein anderes Beispiel ist die Häufigkeit invasiver Massnahmen: Beispielsweise untersuchten Domenighetti et al. die Häufigkeit von Hysterektomien im Kanton Tessin und fanden eine Verdoppelung gegenüber den West Midlands in Grossbritannien bei Fehlen eines signifikanten Unterschiedes in der Mortalität und Morbidität von Uteruskarzinomen [2]. Auch wenn aus diesen Zahlen nicht auf die Qualität der medizinischen Versorgung geschlossen werden kann, weisen sie doch auf bestehende Unterschiede im therapeutischen Vorgehen hin. 


\section{Wechsel im allgemein akzeptierten Vorgehen}

Als wohl bedeutsamster Wechsel im allgemein akzeptierten Vorgehen fordert EBM, der Nutzen medizinischen Handelns sei wenn immer möglich auf der Basis klinisch-epidemiologischer Daten zu beweisen. Der hohe Stellenwert epidemiologischer Daten leitet sich aus der Grundhaltung der EBM ab, die Raspe mit zwei Kernsätzen wie folgt umschreibt: «1) Erfahrung, zumal die eigene klinische Erfahrung, ist trügerisch. Sie ist gefährdet durch die dem Menschen eingeborene Falliabilität, unter anderem in Form unserer Anfälligkeit für Selbst- und Fremdtäuschung. 2) Die besondere soziale Aufgabe der Medizin als Handlungswissenschaft liegt weniger darin, ‘Krankheitsphänomene mit naturwissenschaftlichen Methoden zu analysieren, sondern vielmehr darin, Krankheiten vorzubeugen und Kranke zu heilen und ihnen jede mögliche Linderung zu verschaffen" [3].

Bereits eine einfache Überschlagsrechnung zeigt, wie berechtigt die erste Aussage ist. 1998 wurden in der Schweiz in der ambulanten Praxis 5,4 Millionen Verordnungen für Präparate gegen Infektionskrankheiten gemacht [4]. Bei 13357 praktizierenden Ärzten entspricht dies nach zehn Jahren Praxis um die 4000 Verordnungen zur Behandlung von Infektionskrankheiten, die ein Arzt durchschnittlich überblickt. Selbst wenn er dabei immer das gleiche Antibiotikum verschrieben hätte, wäre sein Erfahrungshintergrund noch viel zu bescheiden für sehr seltene Ereignisse, z. B. einen anaphylaktischen Schock bei Clarithromycin, dessen Häufigkeit weit unter 1 pro 10000 , wahrscheinlich auch weit unter 1 pro 100000 Verordnungen liegen dürfte.

Der zweite Punkt ist grundsätzlicher und begründet, warum pathophysiologische Überlegungen als sekundär betrachtet werden. Die somatische Medizin basiert im wesentlichen auf pathophysiologischen und biochemischen, neuerdings auch molekulargenetischen Denkmodellen: Therapien bestehen in der Regel darin, dass ersetzt wird, was fehlt, weggeschnitten wird, was zu viel ist, und zusammengesetzt wird, was gebrochen ist [5]. Solche Modelle sind zwar pragmatisch, häufig aber auch zu einfach: So folgt der subjektive Nutzen aus der Sicht des Patienten nicht immer der Verbesserung physiologischer Parameter. Als bekanntes Beispiel sei Flecainid erwähnt, welches bei Postinfarktpatienten zwar die Häufigkeit ventrikulärer Extrasystolen verringert, jedoch trotz antiarrhythmischer Wirkung eine erhöhte Mortalität bewirkt.

Die Methode der Wahl, Fehlschlüsse aufgrund falscher oder zumindest unvollständiger pathophysiologischer Modelle zu verhindern, ist die klinischepidemiologische Studie, welche die patientenrelevanten Wirkungen untersucht. Als Goldstandard hat sich der "randomised controlled trial" etabliert: Die Versuchsteilnehmer werden nach dem Zufallsprinzip in Gruppen eingeteilt, die unterschiedlich behandelt werden. Damit wird es möglich, den Erfolg von Therapien $\mathrm{zu}$ vergleichen. Bei methodisch korrekter Durchführung verhindert dieser Studientyp systematische Fehler.

\section{Systematic reviews}

Mit der EBM-Botschaft, therapeutische Optionen möglichst auf der Basis randomisierter kontrollierter Studien zu wählen, ist dem praktizierenden Arzt nur beschränkt geholfen. Nicht vollkommen identische Fragestellungen, unterschiedliche Untersuchungspopulationen oder der Zufall sind Ursachen dafür, dass selbst methodisch gut gemachte Studien in ihrer Aussage divergieren. In der Praxis wird Zeitmangel zudem der wohl wesentlichste Hinderungsgrund sein, immer auf Originalarbeiten zurückzugreifen; Übersichtsarbeiten bilden damit die wichtigste Informationsquelle.

Allgemein herrscht Konsens darüber, dass jede wissenschaftliche Publikation einer detaillierten Beschreibung der zugrundeliegenden Methodik bedarf. Es gibt keinen rationalen Grund, dies nicht auch für Übersichtsarbeiten zu fordern. M. Egger hat die Notwendigkeit dafür an einem Beispiel eindrücklich dokumentiert [6]: Mitchell schrieb 1981 in einer narrativen Review mit dem Titel «Timolol after Myocardial Infarction: An Answer or a New Set of Questions?» im "British Medical Journal»: "... we still have no clear evidence that Beta-Blockers improve long-term survival after infarction despite almost 20 years of clinical trials.» [7] Im gleichen Jahr äusserte sich Hampton im «European Heart Journal» dazu wie folgt: «... it seems perfectly reasonable to treat patients who have survived an infarction with timolol.» [8]

"Systematic reviews» wie beispielsweise diejenigen der "Cochrane Collaboration" haben eine klare Fragestellung, ein dokumentiertes Vorgehen bei der Literatursuche und klare Kriterien für den Ein- bzw. Ausschluss der gefundenen Studien. Die wesentlichen Publikationen $\mathrm{zu}$ einer bestimmten Fragestellung sollen möglichst vollständig erfasst werden; der dafür notwendige Aufwand für die Literatursuche und die kritische Beurteilung aller gefundenen Arbeiten ist gross. Soll der Stand des Wissens weitgehend verzerrungsfrei erfasst werden, ist er jedoch nicht zu umgehen. Falls die statistische Kombination der Studien sinnvoll ist, werden zusätzlich Metaanalysen durchgeführt.

\section{Die Kontroverse um Evidence-based Medicine}

EBM hinterfragt bisher akzeptierte diagnostische Tests und therapeutische Verfahren, um sie bei Bedarf durch effektivere und sicherere zu ersetzen. Das Wort "evidence" wird in der englischsprachigen Bedeutung von "Beweis" verwendet, und es wird damit gefordert, die Richtigkeit von Lehrmeinungen seien prinzipiell zu belegen. Diese klare Absage an die Expertenmeinungen ohne Belege durch Fakten hat EBM wohl am meisten Kritik eingebracht, sahen viele darin doch eine Missachtung ihrer Erfahrung. In Wirklichkeit stellt EBM den Wert klinischer Erfahrung nicht in Abrede, sondern fordert ihre Integration: Nur aufgrund klinischer Erfahrung kann entschieden werden, ob und wie die aus systematischer Forschung abgeleitete 
Evidenz auf einen individuellen Patienten anwendbar ist.

EBM wird auch wissenschaftlicher Positivismus vorgeworfen: Sie verkenne, dass die Realität nicht nur aus quantifizierbaren Phänomenen bestehe und ignoriere wesentliche, quantitativ nicht erfassbare Aspekte der Medizin. Das Argument stimmt, spricht jedoch nicht gegen EBM als Methode; es zeigt lediglich ihre jetzigen Grenzen und damit auch ihren Stellenwert, nämlich als Methode der Wahl zur Beantwortung quantitativer Fragen. In verschiedenen neueren Publikationen ist diese Beschränkung auch bereits kritisch hinterfragt und der Einbezug qualitativer Forschungsmethoden in das EBM-Instrumentarium vorgeschlagen worden.

Auch der Anspruch einiger Exponenten, EBM bedeute einen Paradigmenwechsel, hat zu Polemik geführt. In ihrer eingangs zitierten Beschreibung des Paradigmenwechsels fahren Bortz und Döring fort: «Im weiteren Verlauf des Konfliktes beschränkt sich die Kritik nicht mehr nur auf einzelne Teile des wissenschaftlichen Gebäudes, sondern wird fundamental.» [1] Letzeres trifft für EBM nicht zu, denn die Methodologie, mit der EBM die Probleme angeht, ist Bestandteil des bestehenden wissenschaftlichen Gebäudes. EBM fordert damit vor allem eine Veränderung der praktischen Umsetzung. Um beim Bild zu bleiben: Das Ziel von EBM ist nicht ein neues Gebäude; sie will vielmehr, dass wir dieses Gebäude anders wahrnehmen und vor allem anders darin wohnen.

\section{Verdankung}

Ich danke Daniel Pewsner für seine wertvollen Anregungen und die kritische Durchsicht des Manuskriptes.

\section{Literatur}

1 Bortz J, Döring N. Forschungsmethoden und Evaluation. Berlin: Springer; 1995.

2 Domenighetti G, Lurascho P, Casabianca A, Pedrinis E, Spinelli A. Prévalence et cancer de l'utérus dans la population du canton Tessin. Soz Präventivmed 1984;29:178-9.

3 Raspe H. Evidence-based Medicine: Anlässe, Methoden Probleme. Geburtsh Frauenheilk 1998;58:M21-M25.

4 Pharma Information. Das Gesundheitswesen in der Schweiz. Basel: Pharma Information; 1999.

5 Stam J. Installing Evidence-based medicine in every day practice. Presented at the ECPM Workshop: Evidence-based medicine and the Cochrane Collaboration - a rational base for medical decision making. University of Freiburg, Germany, April 11, 1997.

6 Egger M. Unter dem Metaskop: Möglichkeiten und Grenzen von Metaanalysen. Schweiz Med Wochenschr 1998; 128:1893-901.

7 Mitchel JRA. Timolol after myocardial infarction: an answer or a new set questions? Br Med J 1981;282:1565-70.

8 Hampton JR. Use of beta-blockers for the reduction of mortality after myocardial infarction. Eur Heart J 1981;2:259-68. 